http://jmscr.igmpublication.org/home/ ISSN (e)-2347-176x ISSN (p) 2455-0450

crossref DOI: https://dx.doi.org/10.18535/jmscr/v8i11.25

Journal Of Medical Science And Clinical Research

IGM Publication

An Official Publication of IGM Publication

\title{
Role of Ultrasound in Women with Postmenopausal Bleeding
}

\author{
Authors \\ Bala Bharathi $^{1}$, Amarnath $^{2}$, Kurinjiselvan ${ }^{3}$ \\ ${ }^{1}$ Post Graduate, ${ }^{2,3}$ Assistant Professor \\ Department of Radiodiagnosis, Rajah Muthiah Medical College and Hospital, Chidambaram
}

\begin{abstract}
Background: Objectives of current study were to diagnose benign and malignant causes of abnormal uterine bleeding in postmenopausal women $(P M W)$ by transvaginal ultrasound grey scale imaging and power doppler, thereby minimizing unnecessary interventions in the form of operations and hysteroscopy where sonography findings are normal.

Methods: After obtaining ethical clearance present prospective observational study was conducted from October 2019 to October 2020, to evaluate the endometrium in 75 postmenopausal women (PMW) with bleeding per vagina referred to the department of Radio diagnosis in Rajah Muthiah Medical and Hospital. After applying inclusion and exclusion criterias, the cases were evaluated with ultrasonography both transabdominal (TAS) and transvaginal scan (TVS) with power doppler. Histopathological correlation was done in all cases.

Results: Our study shows majority (37.3\%) of the women with postmenopausal bleed were in the age group of 50-54 years. Most common causes of PMB were atrophic endometrium (41.3\%), Ca cervix (22.7\%), Endometrial carcinoma (5.3\%), Endometrial Hyperplasia (4\%), endometrial polyp (2.7\%), followed by fibroid (1.3\%) in this study. Diagnostic capacities of Endometrial thickness with Endometrial characteristics, Endometrial Echo and Power Doppler was statistically calculated. Sensitivity, specificity, positive predictivity and negative predictivity value of Endometrial characteristics and echo were $85.7 .0 \%, 94.1 \%, 60.0 \%$ and $98.5 \%$ respectively. Sensitivity, specificity, positive predictivity, negative predictivity value of Power Doppler were 100.0\%, 91.5\%, 40.0\% and $100.0 \%$ respectively.

Conclusions: In women with AUB in postmenopausal age ultrasonography (USG) can be considered as an initial imaging modality for diagnosing endometrial diseases. TVS yields high accuracy in differentiating benign from malignant condition when combining endometrial thickness with endometrial characteristics, endometrial echo and power Doppler thus reducing the need for invasive procedure like biopsy.

Keywords: Postmenopausal bleed, Postmenopausal women, Abnormal uterine bleed, Ultrasonography, Transvaginal scan.
\end{abstract}

\section{Introduction}

Post-Menopausal Bleeding (PMB) is defined as abnormal uterine bleeding occurring more than 12 months after the last menstrual period. $^{(1)}$ Postmenopausal bleeding is one of the most common compliant and it could be the only external manifestation of a hidden serious pathology such as endometrial carcinoma. Even without amenorrhea or irregularity, menstruation continuing after the age of 55 years should 
investigated. ${ }^{(2)}$ Excluding endometrial carcinoma is very important in postmenopausal women although the incidence is only 6.96 per 1000 women with postmenopausal bleeding. ${ }^{(3)}$ There are various causes of PMB like endometrial polyps, fibroids, endometrial hyperplasia and atrophic endometrium in addition to endometrial malignancy which accounts for less than $10 \%$. $^{(4)}$ Cancer cervix is also an important cause of PMB in developing countries like India.For planning appropriate therapy, it is important to determine the cause of PMB. Although Endometrial biopsy (EMB) is preferred as the gold standard test to confirm endometrial cancer (5), transvaginal sonography (TVS) is an efficient and acceptable non-invasive method for the early detection of endometrial pathology in postmenopausal women. The thickened endometrium in postmenopausal women is the most important criterion implicating its pathology in USG. ${ }^{(6)}$ But by using endometrial thickness alone will miss, diagnosis of all endometrial cancer. Thin endometrial echos does not exclude type 2 endometrial cancer. So endometrial thickness in TVS when combined with endometrial characteristics, endometrial echo and power Doppler yields high accuracy in differentiating benign from malignant conditions reducing the need for invasive procedures like hystereoscopy and biopsy. The advantage of TVS is that it can be performed with empty bladder and is convenient for the patient and it is suitable for getting more correct gynecological diagnosis, particularly in fatty women with a thick abdomen. TVS is superior to CT and approaches MRI in its ability to provide information about myometrial, cervical and especially, myometrial invasion of endometrial carcinoma. In abnormal uterine bleeding, TVS is the preferred technique for the evaluation of endometrial disorders. ${ }^{(7)}$ Although Endometrial biopsy is the standard test for the diagnosis of endometrial disease among asymptomatic patients, it is invasive, may be uncomfortable, and may not be able to be performed in some patients with cervical stenosis. Ultrasound evaluation is less invasive and more comfortable and can be performed in patients with cervical stenosis. ${ }^{(8)}$

\section{Method}

A 7.5 $\mathrm{MHz}$ transvaginal sector probe was used. The patients with post menopausal bleeding, before examination were asked to empty their bladder. Condom containing the acoustic gel was used to cover the probe. The patient was instructed to lie in supine position, then the scan was performed. In the sagittal plane, the endometrium was imaged. The endometrium was measured from the anterior to posterior myometrial-endometrial junction. If there was fluid in the cavity each layer was measured separately and summed up. Uterine morphology, Endometrial echo pattern and characteristics were studied. If there is suspicion of endometrial carcinoma, the evidence and extent of myometrial invasion were noted. Thereafter, power Doppler study of the endometrium was carried out. Analysis of the power Doppler signals included visual evaluation of number, size, regularity of vessel branching, and presence of areas of densely packed blood vessels (color splashes).

According to power Doppler flow mapping, three different vascular patterns were defined Alcazar et al. $(2003)^{(9)}$ :

1. Multiple-vessel pattern (pattern A): Multiple vessels were found within the endometrium and in the myometrial endometrial interface. This pattern of blood flow was considered as characteristic of endometrial cancer. It reflects the neoangiogenic phenomena that occur within the tumor tissue and the surrounding areas.

2. Single-vessel pattern (pattern B): A single prevalent vessel was identified penetrating the endometrium from the myometrium. This pattern was considered as characteristic of endometrial polyp as this vessel is thought to correspond to the vascularized polyp's pedicle.

3. Scattered-vessel pattern (pattern C): Scanty vessels were identified scattered in the endometrium . This pattern was considered as 
characteristic of endometrial hyperplasia since some studies have shown that angiogenesis is limited in this pathology.

The entire pelvis was additionally examined to rule out any other pathology.

The results of the transvaginal sonogram were interpreted as

- Atrophic endometrium

- Thickened endometrial echo or abnormal endometrial architecture

- Myomas

- Adenomyosis

- Polypoid lesions

- Pyometra

- Endometrial hyperplasia and a suspicious endometrial carcinoma.

- Carcinoma cervix

\section{Exclusion Criteria}

- Hormonal therapy.

- Coagulation therapy.

- Hypothyroidism or hyperthyroidism.

- Liver disease.

- Neglected IUD or Pessary.

\section{Statistical Methods}

In the present study, 75 women with postmenopausal bleeding was prospectively evaluated for the basic characters such as age of menorrhea, and menopause, the associated co morbidities, endometrial thickness, endometrial characters, endometrial echo, power doppler and histopathological findings. The study subjects were described and interpreted in respect of their continuous variables by student independent " $\mathrm{t}$ " test. The categorical variables were interpreted by $\chi^{2}$ (Chi-square) test. The diagnostic tests results between the tests were compared by " $Z$ " test of proportions: The above statistical procedures were under taken with the help of the statistical package namely IBM SPSS statistics-20. The P-values less than or equal to $0.05(\mathrm{P} \leq 0.05)$ were considered as statistically significant.

\section{Results}

In this study, most of the postmenopausal women belonged to the mean age of $56.39 \pm 6.3$ years. The mean endometrial thickness of postmenopausal bleeding patient with malignancy was $21.8 \pm 11.4$ and benign condition was $4.4 \pm 1.8$ $\mathrm{cm}$. Depending on the endometrial echo pattern, TVS diagnosed 10 patients with malignancy, echo was heterogeneous in 6 patients and homogeneous in 4 patients. The postmenopausal women with malignancy, 6 had diffuse irregular endometrial characteristics and 4 with diffuse regular characteristics. In power Doppler, pattern A (Seen in carcinoma) was noted in $26.7 \%$, pattern $\mathrm{B}$ (noted in polyp) in $2.7 \%$, pattern $\mathrm{C}$ (Seen in hyperplasia) in $9.3 \%$ and no abnormal doppler pattern was observed in majority $(61.3 \%)$ of the study population. USG diagnosed atrophic endometrium in $41.3 \%$, Ca cervix in $22.7 \%, \mathrm{Ca}$ Endometrium in $6.7 \%$, endometrial Hyperplasia in $8.0 \%$, Polyp in $8.0 \%$ and thickened endometrium in $13.3 \%$.

Table-1: Comparison of Endometrial Thickness between the Benign and Malignancy

\begin{tabular}{|l|c|c|c|c|c|c|c|c|}
\hline Variable & \multicolumn{2}{|c|}{ Malignancy } & \multicolumn{2}{c|}{ Benign } & Difference & “t” & \multirow{2}{*}{ df } & Significance \\
\cline { 2 - 7 } & Mean & SD & Mean & SD & & \\
\hline ET & 21.8 & 11.4 & 4.4 & 1.8 & 17.4 & 11.717 & 73 & $\mathrm{P}<0.001$ \\
\hline
\end{tabular}

The table-1 compares the endometrial thickness of post menopausal women with malignant and benign condition. The mean ET of malignancy was $21.8 \pm 11.4$ and benign condition was $4.4 \pm 1.8$.
The difference was statistically very highly significant $(\mathrm{P}<0.001)$. 
Fig-1: Comparison of ET between the two groups

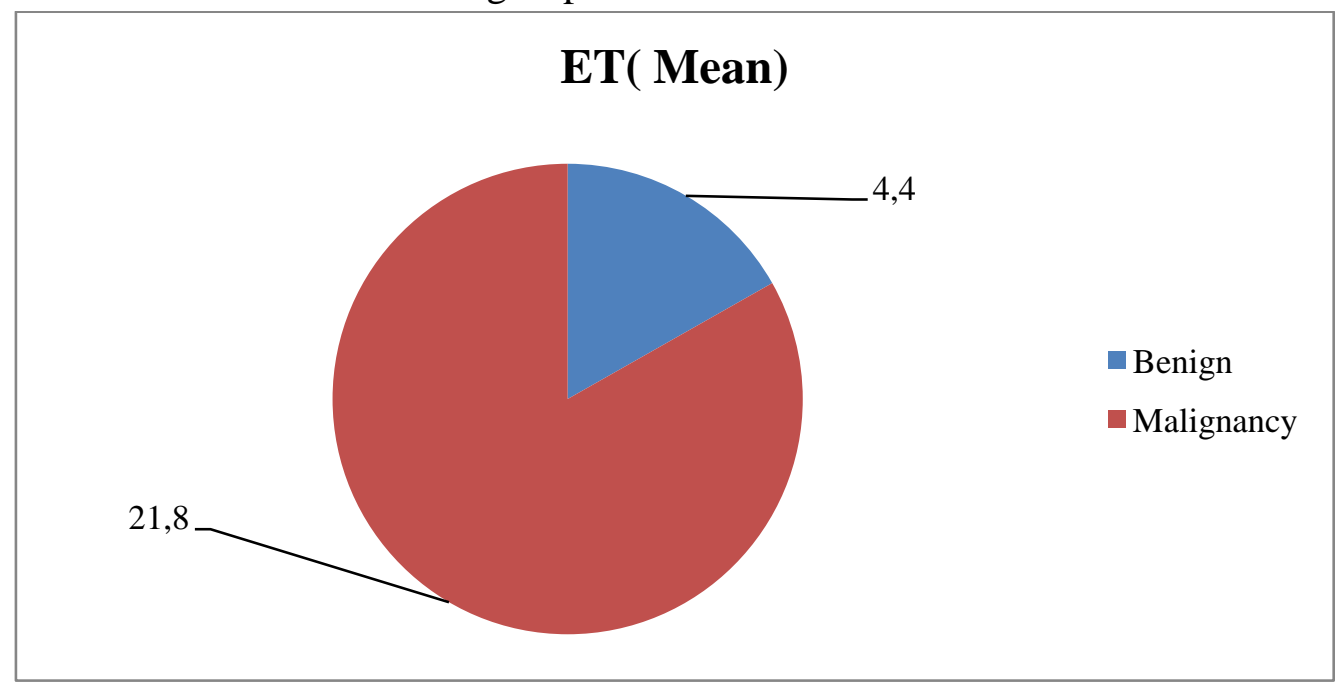

Table-2: Comparison of Endometrial characteristics between Benign and Malignancy

\begin{tabular}{|c|c|c|c|c|}
\hline Endometrial characteristics & Benign & Malignancy & Total & Significance \\
\hline Diffuse irregular & 0 & 6 & 6 & \multirow{5}{*}{$\begin{array}{c}\chi 2=49.038 \\
\mathrm{df}=3 \\
\mathrm{P}<0.001\end{array}$} \\
\hline Diffuse Regular & 12 & 4 & 16 & \\
\hline Focal regular & 7 & 0 & 7 & \\
\hline Thin, Fine & 46 & 0 & 46 & \\
\hline Total & 65 & 10 & 75 & \\
\hline
\end{tabular}

The Endometrial characteristics between the Benign and Malignancy were compared. Diffuse irregular endometrium was noted in 6 patients and diffuse regular pattern was noted 4 patients of study population with malignancy. Patients 65 were diagnosed as having benign condition, out of which 12 had diffuse regular endometrium, 7 had focal regular endometrium and majority (46 patients) had thin, fine endometrium. The Characteristics were statistically significantly differed between the two groups $(\mathrm{P}<0.001)$.

Fig-2: Endometrial characteristics between the two groups

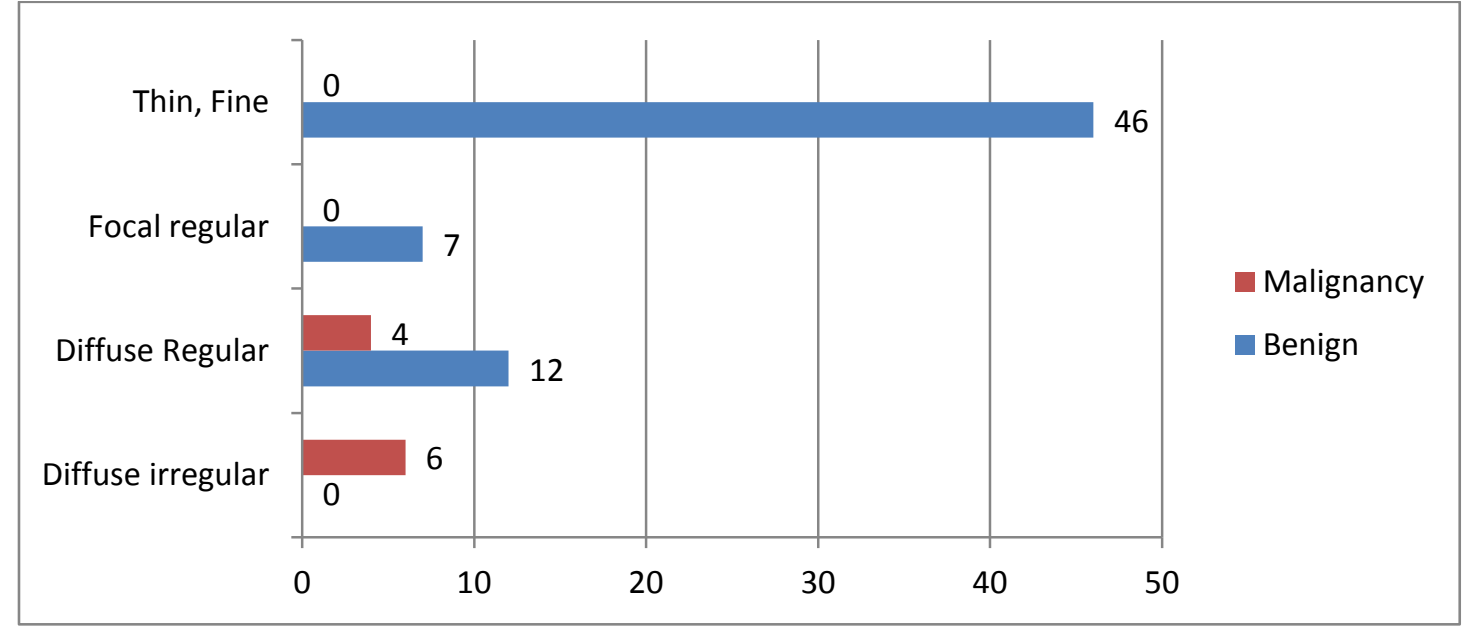

Table-3: Comparison of Endometrial Echo between the benign and malignancy

\begin{tabular}{|l|c|c|c|c|}
\hline Endometrial Echo & Benign & Malignancy & Total & Significance \\
\hline Heterogeneous & 0 & 6 & 6 & \multirow{2}{*}{$2=34.391$} \\
Homogeneous & 65 & 4 & 69 & $\mathrm{df}=1$ \\
Total & 65 & 10 & 75 & $\mathrm{P}<0.001$ \\
\hline
\end{tabular}




\section{JMSCR Vol||08||Issue||11||Page 142-149||November}

The table-3 compares the Endometrial Echo between the two groups. The endometrial echo pattern was homogenous in 65 subjects with benign condition. In patient with malignancy, 6 had heterogeneous and 4 had homogeneous echo pattern. The differences of incidences were statistically significant $(\mathrm{P}<0.001)$.

Fig-3: Comparison of Endometrial Echo between the benign and malignancy

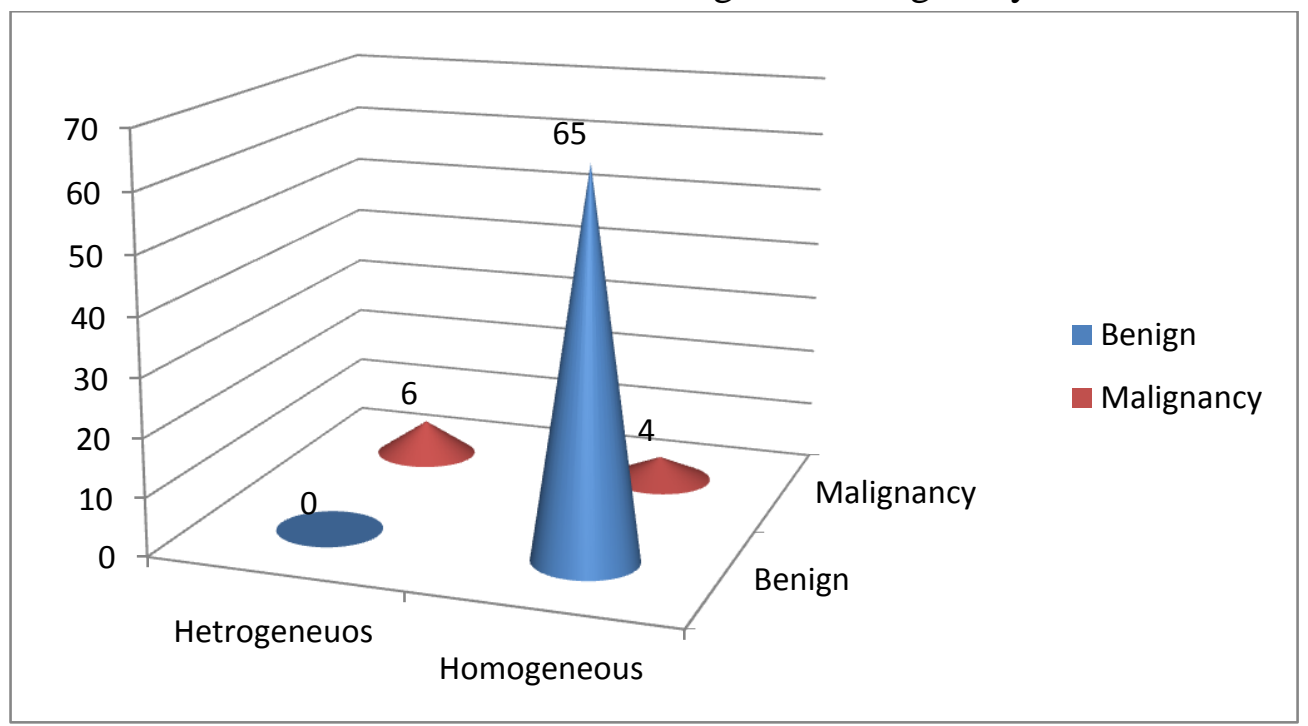

Table-4: Results of Power Doppler

\begin{tabular}{|l|c|c|}
\hline Results & Frequency & \% \\
\hline No abnormal vascular pattern (0) & 46 & 61.3 \\
\hline Disorganised vascular pattern (A) & 20 & 26.7 \\
\hline Single vascular pattern (B) & 2 & 2.7 \\
\hline Scattered vascular pattern (C) & 7 & 9.3 \\
\hline Total & 75 & 100.0 \\
\hline
\end{tabular}

The table- 4 shows the results of Power Doppler. The disorganized vascular pattern (A), single vascular pattern(B), scattered vascular pattern (C) and no abnormal vascular pattern(0), were noted in $26.7 \%, 2.7 \%, 9.3 \%$ and $61.3 \%$ respectively.

Fig-4: Power Doppler results

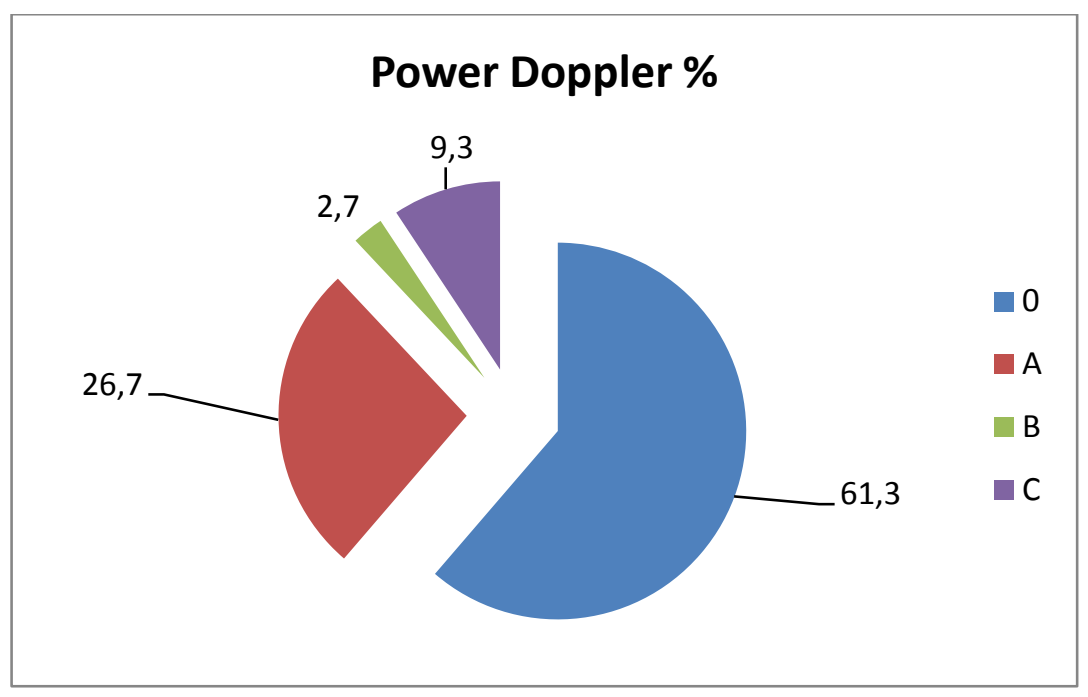


Table-5: Comparison of diagnostic capacity of the tests

\begin{tabular}{|l|c|c|c|c|c|}
\hline $\begin{array}{l}\text { Screening } \\
\text { test }\end{array}$ & Gold Std & $\begin{array}{c}\text { Detected +ve \% } \\
\text { Screening }\end{array}$ & $\begin{array}{c}\text { Detected +ve \% } \\
\text { Gold Std }\end{array}$ & “Z” & Significance \\
\hline ET & $\begin{array}{c}\text { Endometrial } \\
\text { Characteristics }\end{array}$ & 13.3 & 9.3 & 0.775 & $\mathrm{P}>0.05$ \\
\hline ET & Endometrial echo & 13.3 & 9.3 & 0.775 & $\mathrm{P}>0.05$ \\
\hline ET & Power Doppler & 13.3 & 5.3 & 1.716 & $\mathrm{P}>0.05$ \\
\hline
\end{tabular}

The table 5: compares the screening test with Endometrial characteristics, Endometrial echo and power doppler. In predicting positive, the three tests were not statistically significantly differed with endometrial thickness $(\mathrm{P}>0.05)$.

\section{Discussion}

Uterine bleeding is the most frequent symptom, suggesting an endometrial pathology, so each postmenopausal bleeding requires to be investigated early to prevent further complication. Although the incidence of carcinoma among these women is higher, other benign causes of postmenopausal bleeding such as, atrophic endometrium and hyperplasia are also much more common. This study was undertaken to evaluate the uterine cause and various pathological presentation of PMB by non-invasive techniques like TVS in a defined tertiary health care region during a period of one year. This study is a prospective descriptive study. This study shows most of the women belonged to the mean age of the study population was $56.39 \pm 6.3$ years. The mean age at menorrhea was $14.37 \pm 1.17$ years and the mean age at menopause was $50.21 \pm 3.59$ years. The study observed that the common parity status was $2(52 \%)$. The mother with 3 or more children was $30.7 \%$. The parity status of 1 was observed in $13.3 \%$ of the women and only $4 \%$ were nulliparity. Study conducted by Gull B et al $(2001)^{(10)}$ reported that several risk factors including hypertension and diabetes was associated with increased endometrial thickness and abnormality. These factors were compared in this study. Here diabetes mellitus was the common associated co morbidity, observed in $42.7 \%$, hypertension was noted in $21.3 \%$, Obesity was noted in $13.3 \%$ and $22.6 \%$ of the study women had no associated co morbidities. In this study, the mean endometrial thickness of subjects with malignancy was $21.8 \pm 11.4$ and subjects with benign conditions were $4.4 \pm 1.8$. The difference was statistically very highly significant $(\mathrm{P}<0.001)$. Diagnostic capacities of TVS were statistically analyzed in this study. The endometrial echo pattern was homogenous in 65 subjects with benign condition. In patient with malignancy, 6 had heterogeneous and 4 had homogeneous echo pattern. The differences of incidences were statistically significant $(\mathrm{P}<0.001)$. Sensitivity, specificity, positive predictivity, negative predictivity value of Endometrial Echo were $85.7 \%, 94.1 \%, 60.0 \%$, and $98.5 \%$ respectively. The Endometrial characteristics between the Benign and Malignancy were compared. Diffuse irregular endometrium was noted in 6 patients and diffuse regular pattern was noted 4 patients of study population with malignancy. Patients 65 were diagnosed as having benign condition, out of which 12 had diffuse regular endometrium, 7 had focal regular endometrium and majority (46 patients) had thin, fine endometrium. The Characteristics were statistically significantly differed between the two groups $(\mathrm{P}<0.001)$. Sensitivity, specificity, positive predictivity and negative predictivity value of endometrial characteristics were $85.7 .0 \%, 94.1 \%$, $60.0 \%$ and $98.5 \%$ respectively. Power Doppler Pattern A (Seen in carcinoma) was noted in $26.7 \%$, pattern B (noted in polyp) in $2.7 \%$, pattern $\mathrm{C}$ (Seen in hyperplasia) in $9.3 \%$ and no abnormal Doppler pattern was observed in majority $(61.3 \%)$ of the study population. Sensitivity, specificity, positive predictivity, negative predictivity value of Power Doppler were 100.0\%, 91.5\%, 40.0\% and $100.0 \%$. The endometrial thickness with other sonological findings like endometrial characteristics, Endometrial echo and power 
Doppler was compared in this study. The statistical analysis showed that the positive predictive values of the three tests were not statistically significantly differed with endometrial thickness $(\mathrm{P}>0.05)$. The risk of malignancy increased with increasing endometrial thickness, and endometrial thickness was a fairly good predictor of malignancy. Heterogeneous endometrial echogenicity was only slightly superior to endometrial thickness, but it did add information to endometrial thickness. Endometrial thickness and heterogeneous endometrial echogenicity was the best one for predicting malignancy. Adding Doppler variables to this model improved the overall diagnostic performance to greater extent. Hence ultrasound evaluation of the endometrium in women with postmenopausal bleeding starts with a proper measurement of the endometrial thickness.

\section{Conclusion}

Transvaginal sonogram is a simple, non-invasive convenient way to indirectly visualize the endometrial cavity and is useful as a first step diagnostic procedure. Hence ultrasound evaluation of the endometrium in women with postmenopausal bleeding starts with a proper measurement of the endometrial thickness. Heterogenous endometrial echo, irregular myometrial- endometrial border and disorganosed vascular pattern in power Doppler all helps in refining the diagnostic capacity of TVS in detecting malignant conditions. So endometrial thickness in TVS when combined with endometrial characteristics, endometrial echo and power Doppler yields high accuracy in differentiating benign from malignant conditions thus reducing the need for invasive procedures like hystereoscopy and biopsy. This study proves that this diagnostic tool correlates well with the histopathology findings.

In future it appears that the ultrasonogram will continue to take the role of a stethoscope for the Radiologist and Gynaecologist, to see the lining of the uterine cavity and the information obtained seems worthwhile.

\section{References}

1. William J. Butler, David E. Carnovale. Normal and abnormal uterine bleeding. In: William J. Butler, David E. Carnovale, eds. Te Linde's Operative Gynaecology, 10th ed. Philadelphia: Lippincott Williams and Wilkins; 2011: 595-598.

2. Kumar P, Malhotra N. Menopause. In :Jeffcoat's principles of gynecology. $7^{\text {th }}$ ed. Jaypee Brothers Medical Publishers (P) Ltd. 2008 .p.613.

3. Koss LG. Detection of occult endometrial carcinoma. J Cell Biochem. 1995; (Suppl 23):165-73.

4. Yelamanchi Savitha Devi, Talipeni Swapna K. Diagnostic and Operative Hysteroscopy in The Management of postmenopausal bleeding. J Obstet Gynaecol India. 2001 Mar/Apr;51(2):1159.

5. Medverd JR, Dubinsky TJ. Cost analysis model to compare US versus endometrial biopsy in the evaluation of peri- and postmenopausal abnormal vaginal bleeding. Radiology. 2002;222:619-27.

6. Smith-Bindman R, Kerlikowske K, Feldstein VA, Subak L, Scheidler J, Segal $\mathrm{M}$, et al. Endovaginal ultrasound to exclude endometrial cancer and other endometrial abnormalities. JAMA. 1998;280:1510-7.

7. Saksouk FA. Endometrium, carcinoma. eMedicine 2002: 1-29. Available at: http://emedicine.medscape.com/article/403 578-overview. Accessed 12 July 2013.

8. Langlois JP, Turner LF, Aitken PV Jr. Can transvaginal ultrasound detect endometrial disease among asymptomatic postmenopausal patients? J Fam Pract. 2004 Dec;53(12):1003-4.

9. Alcazar JL, Merce LT, Manero MG, Bau S, Lopez-Garcia G. Endometrial volume 
and vascularity measurements by transvaginal 3-dimensional ultrasonography and powerDoppler angiography in stimulated and tumoral endometria: an interobserver reproducibility study. J Ultrasound Med 2005; 24: 1091-1098.

10. Gull B, Karlsson B, Milsom I, Granberg S. Can ultrasound replace dilation and curettage? A longitudinal evaluation of postmenopausal bleeding and transvaginal sonographic measurement of the endometrium as predictors of endometrial cancer. Am J Obstet Gynecol 2003; 188:

401-408. 